\title{
875 AL009, A FUSION PROTEIN AND MULTI-SIGLEC INHIBITOR, REPOLARIZES SUPPRESSIVE MYELOID CELLS AND POTENTIATES ANTI-CANCER EFFECTS
}

Sam Nalle*, Helen Lam, Ling Leung, Spencer Liang, Daniel Maslyar. Alector, South San Francisco, CA, USA

Background Sialic acid-binding immunoglobulin-type lectins (Siglecs) are a family of cell surface receptors expressed predominantly on myeloid cells that function to promote immune tolerance. Tumors increase the expression of sialic acid glycans and co-opt the immunosuppressive effects of Siglecs, driving tumor resident immune cells toward a cancer permissive phenotype. Due to the overlapping expression profile of Siglec family members on myeloid cells, targeting multiple Siglecs is required for robust efficacy. Here, we present data on AL009, an engineered Siglec-9 extracellular domain-Fc fusion molecule that acts as a sialic acid trap and repolarizes suppressive myeloid cells to activate an anti-cancer immune response.

Methods The ability of AL009 to competitively block various Siglec-Fc fusion proteins was assessed using cultured human myeloid-derived suppressor cells (MDSCs). MDSC repolarization was analyzed by flow cytometry. MDSCs were co-cultured with activated CD8 $+\mathrm{T}$ cells with and without exposure to AL009 and functionally assessed for T-cell activation by flow cytometry and ELISA. In vivo tumor models, including the MC38 and E0771 murine syngeneic subcutaneous models and the B16F10 intravenous lung metastasis model, were used to assess AL009 engineered with a murine Fc (AL009m).

Results AL009 competitively blocks the ability of at least 5 inhibitory Siglec family members to bind their corresponding sialic acid ligands. When incubated with MDSCs, AL009 promotes CD163 and CD206 downregulation and induces proinflammatory chemokine secretion, consistent with a repolarization effect. Further, AL009 potently relieves MDSC suppression of $\mathrm{T}$ cells in a co-culture system. In the MC38 and E0771 murine syngeneic subcutaneous tumor models, AL009m inhibits tumor growth as a monotherapy and in combination with the checkpoint inhibitor anti-PD-L1. In addition, AL009m combines with the tumor antigen targeting antibody TRP-1 in the B16F10 intravenous lung metastasis model to reduce tumor burden.

Conclusions AL009 represents a novel approach to targeting the myeloid cell compartment in oncology by directly repolarizing myeloid cells without cell depletion or limiting the targeting to specific suppressive subpopulations. AL009 has the potential to address tumors that are unresponsive or refractory to standard immunotherapies. These data support further development of AL009 in the clinic with IND enabling studies ongoing.

http://dx.doi.org/10.1136/jitc-2021-SITC2021.875 